\title{
Palliative care for patients with pulmonary fibrosis: symptom relief is essential
}

\author{
Kathleen Lindell ${ }^{1}$ and Ganesh Raghu ${ }^{2}$ \\ Affiliations: ${ }^{1}$ Div. of Pulmonary, Allergy and Critical Care, Dorothy P. and Richard P. Simmons Center for \\ Interstitial Lung Disease, University of Pittsburgh, Pittsburgh, PA, USA. ${ }^{2}$ Center for Interstitial Lung Disease, \\ University of Washington, Seattle, WA, USA.
}

Correspondence: Kathleen Lindell, University of Pittsburgh, Div. of Pulmonary, Allergy and Critical Care, Dorothy P. and Richard P. Simmons Center for Interstitial Lung Disease, NW 628, UPMC Montefiore, 3459 Fifth Avenue, Pittsburgh, PA 15213, USA. E-mail: lindellkođupmc.edu

@ERSpublications

Patients with PF-ILD suffer from heavy symptom burden and poor quality of life. Early use of opioids and low dose BDZ in severely ill patients with respiratory comprise may lead to improved symptom control and quality of life for this patient group. http://ow.ly/x50e30mJ6ko

Cite this article as: Lindell K, Raghu G. Palliative care for patients with pulmonary fibrosis: symptom relief is essential. Eur Respir J 2018; 52: 1802086 [https://doi.org/10.1183/13993003.02086-2018].

Symptom burden plagues patients with progressive fibrotic interstitial lung disease (PF-ILD) [1]; this symptom burden, in combination with poor prognosis and uncertainty of disease course heavily impacts quality of life, both for patients and family members [2,3]. Patients with PF-ILD are confronted with a wide range of diagnoses and prognoses; some may live many years with a disease that is responsive to treatment, while others with PF-ILD often have a disease trajectory similar to and/or worse than that of lung cancer $[4,5]$.

Regrettably, treatment interventions targeting the cascades of the pathways of lung tissue response as a consequence of injury, inflammation and fibrosis to date have not lessened this burden. As a consequence, patients with PF-ILD are confronted with progressive symptoms and the overall burden worsens while they endure the suffering in their shortened life span. Indeed, significant emotional sufferings that include depression, anxiety and at times panic episodes are unfortunate expected consequences that confront most, if not all patients with irreversible and progressive fibrotic interstitial lung disease.

Patients suffer common symptoms related to their chronic lung disease which contributes greatly to their morbidity and impacts their quality of life [1]. Symptoms patients most frequently experience include dyspnoea, cough, fatigue, anxiety and depression, and patients experience varying degrees of symptom burden and functional decline as their PF-ILD progresses $[1,2]$. In a review of patients with PF-ILD, the overwhelming majority of patients reported breathlessness (68.2-98\%), cough (59-94\%), heartburn (25-65\%) and depression (10-49\%); this review also found that patients reported constitutional symptoms including sleep disturbances, fatigue, weight loss, and anorexia [1].

It is critical that the clinician is able to make a confident diagnosis of the specific form of PF-ILD as this will be useful to formulate patient-centred, personalised counselling and a plan of care to achieve remission or stabilisation of the disease process, when possible, with identification of symptom burden and repeated assessment throughout the disease course [6]. Delivery of information to patients and caregivers regarding the magnitude of the PF-ILD diagnosis needs to be done in a caring, empathic manner to 
convey realistic hope to the patient and their caregiver, as the delivery of an PF-ILD diagnosis is devastating and forever changes their lives [7]. Early educational programmes can help to increase knowledge of the disease and management options so that patients and their caregivers can have a better understanding of the effect and consequences of these relentlessly progressive diseases $[8,9]$.

Once symptom burden is accurately assessed, there are a number of pharmacological and nonpharmacological therapies available to reduce symptom burden $[6,10,11]$. These include regular counselling, anxiolytic and anti-depressive medications, low-dose narcotics, pulmonary rehabilitation, supplemental oxygen therapy, and early referral to palliative care to alleviate symptom burden and address advance care planning, with the ultimate goal of improving quality of life $[3,12,13]$.

In this issue of the European Respiratory Journal, BAJWAH et al. [14] report the results of a population based longitudinal cohort study of PF-ILD patients starting long term oxygen therapy in Sweden between 2005 and 2014. They examined association of benzodiazepines and opioids on risk of admission to hospital and death in this cohort of 1603 patients and found that benzodiazepines were used by 196 (12\%), and opioids by 254 (15\%) patients when they were initiated on long term oxygen therapy. They reported no association between benzodiazepines and increased admission, but treatment with higher dose versus lower dose benzodiazepines showed association with increased mortality. It is important to note that these patients were advanced in their disease course and some of these patients were receiving benzodiazepines for end of life management of anxiety-related breathlessness. Benzodiazepines reduce the sensation of breathlessness through decreasing the anxiety associated with breathlessness and should be considered at lower doses to decrease symptom burden. Their findings also showed that depressed/anxious patients were much more likely to be prescribed benzodiazepines and opioids. Low or high dose opioids showed no association with increased admission or increased mortality in this cohort. This is the first ever study to look at safety concerns of these pharmacological treatments for symptom management in patients with PF-ILD, and their findings suggest that when benzodiazepines and opioids were used, they were not used until the disease was advanced and the patients were oxygen dependent. These findings support the early use of opioids and low dose benzodiazepines in severely ill patients with respiratory compromise. Improvements in breathlessness management early in the disease course may lead to improved symptom control and quality of life for this patient group.

In essence, patients with PF-ILD, including advanced IPF, suffer from heavy symptom burden and a poor quality of life [15]. This calls for comprehensive symptom management and integrated palliative care concomitant with disease-modifying therapies as soon as the patient is diagnosed with the PF-ILD. The incorrect perception that palliative care is synonymous with end of life care, with no role earlier in the course of ILD, has created a culture of neglect [12]. The stigma of prescribing opioids and benzodiazepines contributes to the symptom burden that patients unnecessarily suffer $[12,16]$.

Early integrated palliative care for patients with lung cancer has shown substantial benefits, such as lower depression scores, higher health-related quality of life, better communication of end of life care preferences, less aggressive care at the end of life, and longer overall survival [17].

We believe that incorporating early palliative care and regular counselling will allow patients with PF-ILD and their caregivers to obtain better and more effective pharmacological and psychosocial interventions to improve quality of life throughout the disease course. Sensitive advanced care planning, and timely patient-centred end of life care with much-needed counselling with patients and their caregivers, as well as appropriate pharmacotherapy to alleviate emotional and physical suffering, must be an integrated part of routine care of patients with PF-ILD.

TABLE 1 Some palliative care studies in interstitial lung disease

Study Manuscript title

BAJWAH and Yorke [18] Palliative care and interstitial lung disease

BARRIL et al. [19] Palliative care in interstitial lung disease: results of a Spanish survey

KALLURI et al. [20] Beyond idiopathic pulmonary fibrosis diagnosis: multidisciplinary care with an early integrated palliative approach is associated with a decrease in acute care utilization and hospital deaths

LINDELL et al. [9] Randomised clinical trial of an early palliative care intervention (SUPPORT) for patients with idiopathic pulmonary fibrosis (IPF) and their caregivers: protocol and key design considerations

Rush et al. [21]

Access to palliative care for patients undergoing mechanical ventilation with idiopathic pulmonary fibrosis in the United States

SHARP et al. [22]

Development of tools to facilitate palliative and supportive care referral for patients with idiopathic pulmonary fibrosis 
Table 1 lists some of the current palliative care studies that are underway. While we are not aware of all clinical studies that are ongoing and/or have been completed, it is hoped that there are and/or will be more clinical studies addressing palliative care in this cohort of patients and provide evidence to enhance further understanding and management of PF-ILD and improve outcomes that are meaningful to these patients.

Conflict of interest: None declared.

Support statement: This work was supported by National Institute Nursing Research Grant \#NR01627. Funding information for this article has been deposited with the Crossref Funder Registry.

\section{References}

1 Carvajalino S, Reigada C, Johnson MJ, et al. Symptom prevalence of patients with fibrotic interstitial lung disease: a systematic literature review. BMC Pulm Med 2018; 18: 78.

2 Garibaldi B, Danoff SK. Symptom-based management of the idiopathic interstitial pneumonia. Respirology 2016; 21: 1357-1365.

3 van Manen MJ, Geelhoed JJ, Tak NC, et al. Optimizing quality of life in patients with idiopathic pulmonary fibrosis. Ther Adv Respir Dis 2017; 11: 157-169.

4 Vancheri C, Failla M, Crimi N, et al. Idiopathic pulmonary fibrosis: a disease with similarities and links to cancer biology. Eur Respir J 2010; 35: 496-504.

5 Raghu G, Chen SY, Yeh WS, et al. Idiopathic pulmonary fibrosis in US Medicare beneficiaries aged 65 years and older: incidence, prevalence, and survival, 2001-11. Lancet Respir Med 2014; 2: 566-572.

Lindell K. Nonpharmacologic therapies in interstitial lung disease. Curr Pulmonol Rep 2018; 7: 1-7.

Swigris JJ. Transitions and touchpoints in idiopathic pulmonary fibrosis. BMJ Open Respir Res 2018; 5: e000317. Morisset J, Dubé BP, Garvey C, et al. The unmet educational needs of interstitial lung disease patients: setting the stage for tailored pulmonary rehabilitatio. Ann Am Thorac Soc 2016; 13: 1026-1033.

9 Lindell K, Nouraie M, Klesen MJ, et al. Randomised clinical trial of an early palliative care intervention (SUPPORT) for patients with idiopathic pulmonary fibrosis (IPF) and their caregivers: protocol and key design considerations. BMJ Open Respir Res 2018; 5: e000272.

10 Lee JS, McLaughlin S, Collard HR. Comprehensive care of the patient with idiopathic pulmonary fibrosis. Curr Opin Pulm Med 2011; 17: 348-354.

11 Kohberg C, Uggerhoj Anderson C, Bendstrup E. Opioids: an unexplored option for treatment of dyspnea in IPF. Eur Clin Respir J 2016; 3: 10.3402/ecrj.v3.30629.

12 Kreuter M, Bendstrup E, Russell AM, et al. Palliative care in interstitial lung disease: living well. Lancet Respir Med 2017; 5: 968-980.

13 Ferrara G, Luppi F, Birring SS, et al. Best supportive care for idiopathic pulmonary fibrosis: current gaps and future directions. Eur Respir Rev 2018; 27: 170076.

14 Bajwah S, Davies JM, Tanash H, et al. Safety of benzodiazepines and opioids in interstitial lung disease: a national prospective study. Eur Respir J 2018; 52: 1801278

15 Yount SE, Beaumont JL, Chen S-Y, et al. Health-related quality of life in patients with idiopathic pulmonary fibrosis. Lung 2016; 194: 227-234.

16 Simon ST, Higginson IJ, Booth S, et al. Benzodiazepines for the relief of breathlessness in advanced malignant and non-malignant diseases in adults. Cochrane Database Syst Rev 2016; 10: CD007354.

17 Temel JS, Greer JA, Muzikansky A, et al. Early palliative care for patients with metastatic non-small-cell lung cancer. N Engl J Med 2010; 363: 733-742.

18 Bajwah S, Yorke J. Palliative care and interstitial lung disease. Curr Opin Support Palliat Care 2017; 11: 141-146.

19 Barril S, Alonso A, Rodríguez-Portal JA, et al. Palliative care in interstitial lung disease: results of a Spanish survey. Arch Bronchneumol 2018; 54: 123-127.

20 Kalluri M, Claveria F, Ainsley E, et al. Beyond idiopathic pulmonary fibrosis diagnosis: multidisciplinary care with an early integrated palliative approach is associated with a decrease in acute care utilization and hospital deaths. J Pain Symptom Manage 2018; 55: 420-426.

21 Rush B, Berger L, Celi LA. Access to palliative care for patients undergoing mechanical ventilation with idiopathic pulmonary fibrosis in the United States. Am J Hosp Palliat Care 2018; 35: 492-496.

22 Sharp C, Lamb H, Jordan N, et al. Development of tools to facilitate palliative and supportive care referral for patients with idiopathic pulmonary fibrosis. BMJ Support Palliat Care 2018; 8: 340-356. 